\title{
Alcohol and Antiretroviral Therapy - A Lethal Cocktail
}

Michelle Schneider $^{1 *}$, Manuela Neuman ${ }^{2,3}$, Matthew Chersich ${ }^{4,5}$ and Charles Parry ${ }^{1,6}$

${ }^{1}$ Alcohol \& Drug Abuse Research Unit, Cape Town, South African Medical Research Council, South Africa

2In Vitro Drug Safety and Biotechnology, MaRS, Toronto, ON, Canada

${ }^{3}$ Departments of Pharmacology \& Toxicology and International Health, Faculty of Medicine, University of Toronto, Canada

${ }^{4}$ Centre for Health Policy, School of Public Health University of Witwatersrand, Johannesburg, South Africa

${ }^{5}$ International Centre for Reproductive Health, Ghent University, Ghent, Belgium

${ }^{6}$ Department of Psychiatry, Stellenbosch University, Cape Town, South Africa

\begin{abstract}
Background: Alcohol plays a role at the different points in the natural history of HIVIAIDS: This article focuses on the health implications of harmful alcohol consumption in the era of antiretroviral therapy.

Aim: To explore the role of alcohol in HIV disease progression, in order to improve HIV patient management and overall better HIV prognosis.

Methods: An examination of studies pertaining to the behavioral, biological and bio-chemical aspects of alcohol consumption on the pathogenesis of HIV.

Findings: Alcohol consumption impacts on HIV progression resulting in increased morbidity and mortality. Alcohol consumption reduces compliance with ARV regimens, resulting in additional premature mortality. Both alcohol and HIV modulate innate and adaptive immunity and alcohol consumption for HIV-positive individuals increases the likelihood of viral replication and leads to increased susceptibility to contract opportunistic infections and other co-morbid conditions. The situation is further compounded by drugs used for the treatment of the opportunistic infections and other co-morbid conditions and their potential interactions with alcohol. The liver also metabolizes both alcohol and ARV drugs and alcohol-related liver toxicity results in compromised liver function with ARVs not working optimally and an increased risk of serious toxicity from antiretroviral therapy.

Discussion: Very diverse measures of alcohol consumption have been used in studies on interactions between alcohol and HIV, making it difficult to compare studies and draw definitive conclusions. It is essential to acquire clear evidence-based guidelines on alcohol consumption for HIV-positive patients and their health-care providers. The variables alcohol, HIV and ART and their myriad interactions have not been clearly delineated. The multiple effects from HIV, alcohol and ART may compound each other, making it difficult to disentangle presenting adverse reactions and specifically the associations with alcohol. Furthermore findings in this arena are particularly relevant for prevention and treatment of HIV in countries such as South Africa that have high HIV and alcohol health burdens and have committed to an extended ARV rollout.
\end{abstract}

Keywords: HIV; AIDS ARV; HAART; Alcohol; Pharmacology; Immunology

Abbreviations: HIV: Human Immunodeficiency Virus; AIDS: Acquired Immune Deficiency Syndrome; ARV: Antiretroviral; ART: Antiretroviral Treatment; HAART: Highly Active Antiretroviral Treatment; PLWA: People Living with AIDS; MSM: Men who have Sex with Men; NRTI: Nucleoside Reverse Transcriptase Inhibitor; PI: Protease Inhibitor; RNA: Ribonucleic acid; AUDIT: Alcohol Use Disorders Identification Test; DUDIT: Drug Use Disorders Identification Test

\section{Introduction}

The estimated 9.5 litres for per capita pure alcohol consumption per annum in South Africa (SA) is not high in global terms. However, when taking into account the high levels of abstention, it has one of the highest levels of per capita consumption per drinker globally [1].

South Africa also has the highest number of people infected with HIV in the world. UNAIDS [2] estimated that in 2009 South Africa (SA) had 5.7 million people living with HIV. The current South African criterion for initiating antiretroviral therapy (ART) is a CD4 count of 350 cells $/ \mu \mathrm{L}$ [3]. The country now has the largest ART programme in the world, with an estimated 1.4 million people on ARVs in 2011 [4]. Although this is a large number, the figure still indicates a substantial unmet need for ART in South Africa.
The afore-mentioned HIV and alcohol statistics for SA are alarming on their own. Increasingly as the effects of alcohol misuse on poor prognosis for HIV become known there is a need to predict the poor health outcomes resulting from this hazardous mix. This will result in improved management regarding alcohol consumption for HIV patients, particularly those on antiretroviral drugs (ARVs).

Alcohol plays a role at the different points in the natural history of HIV/AIDS: Initially, on contracting the disease, then in the period prior to HIV-infected persons requiring ART and finally when receiving ARV drug treatment. This article addresses the last-mentioned stage,

*Corresponding author: Michelle Schneider, Alcohol \& Drug Abuse Research Unit, Cape Town, South African Medical Research Council, South Africa, Tel: +27 21-7022165; Fax: +27 21-9380-0324; E-mail: mschneider@global.co.za

Received October 19, 2011; Accepted January 25, 2012; Published January 29, 2012

Citation: Schneider M, Neuman M, Chersich M,Parry C (2012) Alcohol and Antiretroviral Therapy - A Lethal Cocktail. J AIDS Clinic Res S1:005. doi:10.4172/2155-6113. S1-005.

Copyright: (c) 2012 Schneider M, et al. This is an open-access article distributed under the terms of the Creative Commons Attribution License, which permits unrestricted use, distribution, and reproduction in any medium, provided the original author and source are credited. 
the health implications of harmful alcohol consumption in the era of ART.

The advent of ART has meant that HIV, though an infectious disease, with life-long treatment can be considered a chronic condition. Overall ART has led to significant reductions in HIV morbidity and mortality. In terms of morbidity, the use of ARVs has been associated with several toxicities that limit their success. Adverse reactions from ART are further complicated by alcohol use. For example, a review by $\mathrm{Oh}$ et al. [5] notes that dyslipidemia is observed with increasing frequency among HIV patients, due to HIV and the ARVs. They suggest that secondary causes of dyslipidemia, such as alcohol consumption, should be identified and treated accordingly. Furthermore, alcohol consumption is causally linked to non-adherence to antiretroviral treatment that in turn causes an increase in HIV/AIDS mortality. Gmel et al. [6] has determined the magnitude of detrimental life expectancy outcomes for those on ARV treatment combined with alcohol consumption, based on the impact of alcohol consumption on adherence to antiretroviral medication. He found that for the African Global Burden of Disease, this effect of alcohol on HIV/AIDS in the African Global Burden of Disease regions ranged from $0.03 \%$ to $0.34 \%$ for men and from $0 \%$ to $0.17 \%$ for women, depending on region and age category. Overall these figures reflect a statistically significant detrimental effect of alcohol consumption on life expectancy for people on ARVs.

As shall be discussed below, the effects of alcohol consumption on individuals are determined by volume, pattern of use and type of drink consumed. Furthermore, its effects (and those of ARVs) vary by the genetic make-up of the patient, their weight and gender [7]. Very diverse measures of alcohol consumption have been used in studies on interactions between alcohol and HIV, making it difficult to compare studies and draw definitive conclusions on the effect of alcohol on the pathogenesis and natural history of HIV infection.

The accurate measurement of alcohol consumption is beset by technical difficulties as well as beliefs and social practices associated with alcohol consumption. Social-desirability biases [8] related to alcohol consumption are possibly compounded with the perceived stigma of being HIV-positive. Hahn et al. [8] compared self-reported alcohol consumption with a biomarker of heavy alcohol consumption in persons initiating antiretroviral therapy in Uganda. Results suggested marked under-reporting among those claiming abstinence and in heavy drinkers. In a developing country such as Uganda, alcohol consumption also comprises home-brews, with varying alcohol content and drink size, making accurate calculations of alcohol consumption very difficult. Furthermore, it is often difficult to ascertain the quantity of alcohol consumed, as drinking may be communal with shared drinking receptacles.

The different drinking patterns reflect how people drink, rather than the quantity consumed. Different drinking patterns have implications for health outcomes. Patterns of drinking scores (PDS) can be determined by weighting different components. These include, for example, drinking in public places or the proportion of drinking events that result in intoxication or binge drinking, that is, drinking excessively per drinking occasion. South Africa scores high for the various components (4 out of 5) and consequently has a high risk drinking pattern [9]. The PDS for different countries tend to be strongly associated with the alcohol-attributable burden of disease, with the higher the score, the greater the alcohol-attributable burden of disease [9].
A study by Míguez-Burbano et al. [10] examined the choice of alcoholic beverage on ART outcomes. This study highlights that it is not only volume and patterns of drinking that impact on health outcomes but also the type of alcohol consumed is of relevance for health outcomes. Adherence to ART was controlled for in this cohort study for a population initiating ART therapy. The results indicated that liquor consumption that included gin, vodka and whiskey, as opposed to consuming wine and beer resulted in poorer viro-immune outcomes, after initiation of ART.

The role of alcohol consumption in HIV transmission, acquisition and the stages of the disease course is an important one and needs to be clearly delineated. This can be achieved to some extent by examining the behavioural and biological aspects of alcohol consumption on the pathogenesis of HIV. The focus on the biological aspects of alcohol consumption on the human immune system include viral replication, co-morbid conditions, organ disease and poor efficacy of antiretroviral treatment regimens. Furthermore, it needs to be emphasized that many of these poor health outcomes are preventable.

\section{Effects of Alcohol Use on Sexual Behaviour and Adher- ence to Antiretroviral Treatment}

Though broad economic and cultural factors largely determine the spread of HIV, increasingly there is evidence $[11,12]$ that the use of alcohol is also a key determinant of HIV transmission. A study by Braithwaite [13] found that alcohol use among PLWA is higher than in the general population. In both HIV-negative and in infected people, alcohol use is associated with high risk sex $[14,15]$. ARV medication may make a person feel better, have increased libido and be less concerned about HIV infection and transmission; being on ART is consistently associated with greater sexual risk taking that includes lower condom use [16]. A study on a group of HIV-positive MSM on treatment, for example, recorded a self-reported decrease in condom motivation. One factor associated with this was increased alcohol/drug use. In addition sexual risk appeared to be related to beliefs about how treatment affects the transmissibility of HIV. Race, socioeconomic status, alcohol/drug use, mental health and viral load were also associated with treatment beliefs [17]. The very efficacy of the treatment may lead to complacency and less motivation to adhere to antiretroviral drug regimens [18].

When an individual infected with HIV engages in unprotected sex, possibly as a result of alcohol consumption, this may result in re-infection (super-infection). This may lead to a more rapid disease progression and the development of treatment resistant recombinant viruses [19]. Engaging in unprotected sexual intercourse can result in the acquisition of other STIs which in turn place individuals at risk for both contracting and transmitting HIV [20]. Co-infection with STIs can lead to peaks in viral load which can increase the probability of infection. For unprotected heterosexual intercourse and alcohol consumption, the probability of HIV transmission is further increased as moderate to heavy alcohol consumption is positively correlated with vaginal shedding of HIV for patients on ART, even after adjusting for adherence [21].

High levels of adherence, in the order of $95 \%$, are vital for ARVs for HIV to be effective [22]. Besides the resulting low efficacy of the drugs, poor compliance with drug regimens can lead to the development of viral resistance [23]. This has serious ramifications not only for the individual concerned but can also lead to the spread of drugresistant HIV mutations in the population. The role of alcohol in ARV adherence is ubiquitous. For example, even paediatric adherence to ARVs is negatively affected by the use of alcohol by the caregiver [24]. 
A meta-analysis by Hendershot et al. [25] aggregated studies on the alcohol-adherence association. In the combined analysis, drinkers had nearly double the risk of non-adherence compared with abstainers (or those who drank relatively less). In addition, a dose-response relationship indicated that the greater the consumption of alcohol the worse the progression of the disease. Alcohol intake has also been documented as negatively associated with adherence to ARVs in South Africa [26]. This is consistent with other research findings in low-income settings such as India, [27] West Africa, [28] and Botswana [18]. Van Geertruyden et al. [29] postulated that the interaction between HIV and alcohol use may even have a greater public health impact for subSaharan Africa (SSA), exceeding that based on the studies reviewed by Hendershot et al. [25], given that the 40 studies included in the metaanalysis were for countries with an HIV prevalence of less than $1 \%$.

\section{Impact of Alcohol on HIV Disease Progression}

In addition to the association of alcohol with the behavioural variables of unprotected sexual intercourse and poor adherence to HIV medication, there are other more distinctly biological factors relating to alcohol consumption and ART. These biological interactions provide an evidence-base for advice and other interventions to alter alcohol consumption for patients receiving ART.

Diaz et al. [30] demonstrated that moderate consumption of alcohol, particularly of alcoholic beverages containing antioxidants may protect immune cells from damage. Hence, the health impact of certain types and patterns of alcohol consumption generally and more specifically for HIV positive individuals may be beneficial. However, the effect of alcohol on human health and welfare is overwhelmingly negative, certainly at a population level, and similarly, the impact of alcohol on the acquisition, transmission and natural course of HIV is considered to be detrimental.

The negative effect of alcohol consumption on HIV prognosis may be a more general one; not targeting specific systems in the body. According to Watzl et al. [31] alcohol is toxic for the host defence system. In addition, excessive consumption can induce malnutrition which impacts negatively on immune-competence. Alcohol consumption has a detrimental effect on many organs and tissues in the human body, which impacts on HIV pathogenesis. In particular, infection with HIV results in the depletion of mucosal CD4+ lymphocytes. Balagopal et al. [32] hypothesized that both alcohol and HIV may accelerate liver disease through microbial translocation (disruption of gut epithelial integrity and increased mucosal translocation of bacteria and bacterial products). However, the authors point to a more plausible explanation, namely, that microbial translocation is both a cause and an effect of liver disease progression and systemic immune activation.

In addition, animal and human studies demonstrate that alcohol consumption has detrimental effects on both the innate and the acquired immune systems [33]. The CD4 cell count indicates the strength of an individual's immune system. The viral load is a measure of the level of the HIV virus in the patient's blood and is used to monitor the effectiveness of ART. The CD4 count and the viral load together predict HIV progression; they co-vary inversely. The higher the viral load and the lower the CD4 cell count, the faster the HIV patient contracts infections and opportunistic diseases and reaches the final stages of AIDS and ultimately death. A study by Samet et al. [34], concluded that alcohol consumption was associated with lower CD4 counts and higher RNA (ribonucleic acid) counts; compliance was a control variable for those on ART with a history of alcohol problems. In a later study by Samet et al. [35] heavy alcohol consumption, compared with abstinence, was associated with lower CD4 cell but not with higher viral load, for patients not receiving ART. Heavy alcohol consumption accelerates HIV disease progression for those not yet on ART. The effect of alcohol on CD4 cell count seems to be independent of ART [36] and alcohol intake does not appear to directly impact on HIV viral load. The effect of alcohol on viral load instead seems to be via reduced ART adherence [37].

Many of the effects of alcohol consumption on innate (nonspecific) immune response are dose dependent, with acute or moderate use associated with attenuated inflammatory responses, and heavy ethanol consumption linked with the augmentation of inflammation [38]. Acute alcohol exposure suppresses the production of proinflammatory response, including tumor necrosis factor (TNF)- $\alpha$ and IL-1 $\beta$ in macrophages in the lungs and in the white blood cells, important in immunity [38]. Chronic alcohol consumption, in contrast leads to increased levels of pro-inflammatory response; the implication of this is that there are more cells for the HIV to attack throughout the body and at key transmission sites.

The in vitro, specific immuno-modulatory effects of ethanol (alcohol) have been documented: Alcohol consumption attenuates adaptive immune response reducing the number of CD4+ and CD8+ $\mathrm{T}$-cells as well as reducing innate immunity natural killer (NK) cells and cytokine production related to adaptive immunity [39]. Impaired host defence after alcohol ingestion is linked to a combination of decreased inflammatory response, altered cytokine production and abnormal reactive oxygen intermediate generation [40]. Szasbo [40] states that a key element in the alcohol-induced decrease in cell-mediated immunity is decreased antigen presenting cell function. Alcohol inhibits antigen presentation required to regulate the activity of T-and B-cells, a key step in the initiation of the adaptive immune response.

Both alcohol and HIV modulate innate and adaptive immunity by disrupting cytokine and chemokine signalling, depleting crucial immune cell populations, inhibiting maturation of key immune effectors such as dendritic cells, and impairing CD4+ Thelper function, and antiviral cytotoxic T cell activity [41]. From these examples it can be inferred that alcohol consumption, via its impact on the immune system, alters the pathogenesis of HIV.

\section{Effect of Alcohol on the Liver in an HIV-Infected Person}

\section{The liver}

Liver disease in HIV-infected persons is widespread. Alcoholic injury is the most common toxic disorder of the liver. Long-term harmful use of alcohol results in a range of liver diseases including cirrhosis and hepatocellular carcinoma [42]. In the liver, persistent cytokine secretion results in chronic inflammation leading to conditions such as hepatitis, fibrosis, and cirrhosis. Cytokines also regulate a process known as programmed cell death, or apoptosis, which is in part responsible for alcohol-induced destruction of liver tissue [43].

The liver metabolizes alcohol almost exclusively via three main metabolic pathways: microsomal ethanol oxidizing system, alcohol dehydrogenase and catalase-peroxidation reactions [44]. The mechanism of ethanol-induced hepatotoxicity is due to redox changes generated in its oxidation via the alcohol dehydrogenase $(\mathrm{ADH})$ pathway that leads to the formation of the toxic metabolite acetaldehyde. Lieber [45] discusses the role of acetaldehyde toxicity and alcohol-induced oxidative stress. The latter, includes glutathione (antioxidant) depletion. 
Hepatocytes are the main functional cells of the liver with diverse functions that include detoxification, modification, and excretion of exogenous and endogenous substances. Hepatocytes are rich in mitochondria, which are specifically affected by ethanol intoxication [46]. Nucleoside analogues, with antiretroviral activity cause specific mitochondrial toxicity [47].

\section{Bio-chemistry of the liver}

The liver is the central organ that metabolises ARVs via the human cytochrome enzyme P4503A4 CYP3A4. This enzyme system metabolises most of the ART drugs. Ethanol is also oxidized in the liver by an ethanol-inducible cytochrome P-450 (CYP). This reaction, however, is associated with the release of free radicals which can cause lipid peroxidation and liver injury, including mitochondrial damage. Mitochondrial damage in turn exacerbates oxidative stress [48].

The fact that alcohol degradation utilizes the P450 enzyme system in the liver and that this is also the most common metabolic pathway for ARV medication points to possible problematic pharmacokinetic and pharmacodynamics interactions $[49,50]$.

The pharmacological interactions of ARV medication with alcohol include the pharmacodynamics, the study of what a drug does to the body, and pharmacokinetics, the study of what the body does to a drug. Two drugs may have interactions in the body ranging from both remaining unchanged; plasma drug concentration of both drugs increasing or decreasing either in the same or opposite directions; or one being unaffected while the other changes. This change may be in either direction, that is, it increases or decreases the effect. When several drugs are involved the potential number of drug-on-drug interactions increases. There may be additive and synergistic effects of alcohol on ART, in addition to the effects of alcohol on other medications for HIV-related and other health conditions, or alcohol can decrease the effect of ART [51]. The drug-drug interactions are complex and difficult to predict. The multiple effects from HIV, alcohol and ART may compound each other, making it difficult to disentangle presenting adverse reactions and the specific associations with alcohol. Adding alcohol essentially amounts to adding another drug to a potentially toxic brew.

These interactions have the potential to complicate HAART therapy in HIV-positive patients who consume alcohol. Alcohol aggravates toxicities and impacts on plasma drug concentration by either increasing it or decreasing it. Enzymatic induction is a process, whereby a drug stimulates more enzyme proteins to be formed. This can enhance oxidative metabolism and reduce the serum concentrations of drugs; whereas enzyme inhibition involves competition with another drug for the enzyme binding site i.e. reduced drug clearance and extending the serum half-life of a drug [52].

Alcohol and other drugs are metabolized by the same enzymes that transform the ARVs into the associated toxic metabolites. For example; Phase 1 enzymes alcohol dehydrogenase and P450 are further broken down by Phase 2 enzymes such as glutathione. The liver detoxifies the toxic metabolites via the glutathione system [53]. When glutathione is depleted due to rapid consumption of alcohol there is no more left to detoxify the ARVs and as a result the toxic metabolites of the ARV and of alcohol accumulate in the liver inducing organ damage. In addition HIV-infected individuals may have a lower level of glutathione even if they are not drinkers or on ART [54,55]. An additional insult produced by ARVs or alcohol or their combination might lead to an adverse drug reaction for HIV-positive individuals.

\section{Hepatits}

Co-infection by HIV and hepatitis viruses, (HCV and HBV), is a frequent condition in alcohol dependence and intravenous drug use (IDU) as there is a common transmission pathway [12,35,56]. Moreover alcohol and illicit drugs contribute to reduce cellular capacity to fight viral infections [57]. Depending on the risk group, it is estimated that approximately 30 to 80 percent of patients with HIV have concomitant hepatitis $\mathrm{C}$ and approximately 5 to 10 percent have chronic hepatitis $\mathrm{B}$. End-stage liver disease is a major cause for inpatient admissions and mortality in alcoholic co-infected individuals [58]. HIV and HCV coinfected individuals develop liver diseases earlier and more severely than mono-HIV-infected individuals [42]. This is due to the fact that HCV virus has a higher rate of replication in immune deficient hepatocytes.

\section{Interactions between Alcohol and Antiretroviral Drugs and Other Drugs}

Two ARV drugs, Non-Nucleoside Reverse Transcriptase Inhibitors (NNRTIs) and Protease Inhibitors (PIs) are susceptible to increased liver metabolism resulting from the effect of alcohol on the CYP3A4 enzyme system. The resulting sub-therapeutic drug levels in patients taking ARVs and abusing alcohol leads to suboptimal viral control [51]. Alcohol interferes with protease inhibitor metabolism [36] and non-nucleoside reverse transcriptase inhibitors (NNRTs), increasing the serum levels of the drugs and their toxicities [42].

The interaction of ARVs and alcohol may also lead to adverse reactions such as toxic epidermal necrolysis, hypersensitivity syndrome reaction and liver failure [42]. An adverse drug reaction (ADR), in this context is considered to be a clinical consequence due to alcohol intake before, concomitant to or immediately after ARV intake.

Studies examining the association between alcohol consumption and the risk of ARV drug toxicity may have a low proportion of alcohol abusers and small sample sizes may make it difficult to demonstrate any interactions. Furthermore sub-populations on ART may have different alcohol induced adverse reactions. Recent research highlighted an interaction between race and alcohol's effects on dyslipidemia in people receiving ART: alcohol elicits different changes in lipid profiles depending on ethnicity [59].

It should also be noted that many of the studies pertaining to the pharmacological effects of alcohol are from laboratory or animal studies, examining biological and molecular changes from alcohol. These in vitro type studies, while important, cannot definitively show how alcohol impacts on the functioning of the various organ systems of the human body.

\section{Co-morbid conditions}

Most individuals on ARVs take multiple drugs to avoid coinfection, particularly for opportunistic infections associated with HIV/ AIDS. Generally, adverse drug reactions occur at a greater frequency in HIV-infected individuals [60]. These adverse drug interactions may increase as HIV patients on ART age and with the development of other concomitant chronic diseases and their prescribed medications [61]. For example, anti-TB treatment is more difficult in HAART patients as both anti-TB antibiotics and anti-HIV PIs are metabolized by the cytochrome P450 enzyme system, resulting in altered pharmacokinetic profiles for both classes of drugs. For example, blood levels of amprenavir, lopinavir/ritonavir and atazanavir, (PIs), are lowered by anti-TB antibiotics, with rifampicin and rifabutin in particular 
lowering the blood concentrations of nevirapine, efavirenz and delavirdine to sub-therapeutic levels, the consequence of which may be HIV-resistance [62]. To add to the mix, for chronic, heavy drinkers the enzyme Cytochrome P450 2E1 also metabolizes any alcohol consumed.

The hepatotoxicity of other drugs used to prevent HIV opportunistic infections such as sulphametaxasole, requiring activation of enzymes of the endoplasmic reticulum is enhanced after chronic alcohol consumption [60].

Besides the ARVs and the drugs used to treat opportunistic infections and other co-morbid conditions with the potential additive or synergistic adverse effects that may result from the simultaneous consumption of alcohol with these; there is a range of concurrent, necessary medications that may also have adverse health outcomes linked to alcohol use. These include over-the-counter medication, such as painkillers or anti-inflammatories for injuries, recreational drugs, drugs to treat addiction and other mental health conditions as well as homeopathic and herbal remedies.

\section{The simultaneous intake of other drugs}

CYP2E1 activates other drugs to toxic radicals thereby explaining the increased vulnerability of the heavy drinker to commonly prescribed drugs [60]. Conversely, administration of ethanol with the respective drug inhibits biotransformation of the drug since there is competition for the enzyme that block biotransformation of the drug (Neuman 2003).

\section{ARVs, addictive drugs and alcohol}

Substance abuse is common among HIV positive persons. There are interactions for ARVs and treatment for addiction: For example, the NNRTIs, nevirapine and efavirenz were associated with symptoms of methadone withdrawal [63].

\section{Treatment for alcoholism}

There is also the possible interaction of ARV drugs, with the pharmacological treatment for alcohol use problems, these include, naltrxone, disulfiram or acamprosate [42]. For example, disulfiram is used to treat alcoholism as it inhibits acetaldehyde dehydrogenase, causing the accumulation of acetaldehyde after alcohol ingestion, which results in nausea, flushing, headaches, and hypotension [64]. Due to the risk of hepatotoxicity, the use of this agent has to be monitored carefully, especially when on ARVs [42]. The pharmacokinetic parameters of zidovudine are not influenced by naltrexone [65]. Abacavir and disulfiram should not be mixed in HIV-patients who consume alcohol as both drugs inhibit acetaldehyde dehydrogenase [66]. Furthermore, a liquid formulation of lopinavir/ritonavir containing alcohol should not be administered with disulfiram due to the potential of developing disulfiram-like reactions [64].

\section{Other mental health conditions}

Alcohol affects other mental health conditions, for example, depression: Sullivan et al. [67] examined the impact of alcohol use on depressive symptoms in HIV positive patients and they found that alcohol problems are associated with more depressive symptoms. Furthermore, Leserman [68] provided evidence that trauma and depression negatively affect HIV disease progression in terms of markers such as decreases in CD4 T lymphocytes and increases in viral load.

\section{Traditional medicines}

Chopra et al. [69] interviewed nearly 200 people in three South African locations where antiretroviral therapy had been available for at least six months. The majority of subjects were HIV-positive and over half of those were on ART. Overall, they found more enthusiasm for traditional medicines and dietary supplements than for ART, and subjects seemed to view ART as just a variation of these treatments.

Particularly in South Africa where the use of traditional herbal medicines is widespread and coupled with an extensive ARV rollout programme there is concern about potential herbal remedy and drug interactions, possibly exacerbated by alcohol consumption. A SA study, by Mogatle et al. [70] sought to evaluate the effect of the African potato (AP), on the pharmacokinetics of an ARV drug, efavirenz. The AP is used as an immune system booster. Pharmacokinetic data generated during this study indicated that AP did not significantly alter the pharmacokinetics of efavirenz. However, alcohol consumption by study participants was forbidden prior to and during the study. There is a need for clinical studies of the interaction of herbal remedies with ART and alcohol.

\section{Alcohol consumption and ART - common beliefs}

There is a commonly held belief not to mix medication and alcohol [71]. Based on the potential of developing adverse drug reactions and drug-drug interactions between HAART and alcohol, HIV patients generally believe that the two should not be mixed together [72]. Many patients stop taking ARVs when binge drinking [72]. Fitzgerald et al. [73] document alcohol as a barrier to treatment uptake in SA. He reports that rural men initiating ARV treatment have selfimposed delays to enrolment while trying to stop or reduce alcohol use. However, a qualitative study by Schaham et al. [74] showed that among HIV-infected individuals, alcohol is not perceived to affect HIV progression and health service providers rarely discuss alcohol use with patients. An earlier study by Sankar et al. [75] however, found that most patients (85\%) believed that alcohol and ART do not mix; with more than half of the clinicians stating that alcohol and ART should not be taken together. In the Sankar et al. study [75] of African Americans, three consumption groups, i.e. light, moderate, and heavy had differing views on drinking alcohol and ART; with $64 \%$ of light and $55 \%$ of moderate drinkers foregoing ART when drinking compared to $29 \%$ of heavy drinkers. It appears that these patients made a conscious decision not to drink and take ARVs simultaneously and the noncompliance with medication was not the result of being inebriated.

\section{Discussion}

Alcohol use may result in risky sexual behaviour for contracting and transmitting HIV, additional poor health outcomes once a person is HIV-infected, and accelerated HIV disease progression. According to Hahn et al. [76], the degree to which alcohol use impacts HIV progression has not been fully explicated. Alcohol use as a co-factor in risky behavior impacts on progression via non-compliance to ART. Biological mechanisms are also involved that require further study: The acceleration of the course of HIV is largely via a compromised immune system response and more co-morbid conditions. However, Pandrea et al. [51] state there is little knowledge on the interactions between alcohol and HIV induced immunological changes.

The treatment of HIV/AIDS is complex and adverse effects of alcohol on ART cause many additional health problems. It has been shown that the myriad of pharmacologic consequences as well as the 
Overall, the harmful consumption of alcohol impacts on the length of life as well as costs of health care for the AIDS patient. There is increasing evidence that no level of alcohol consumption is safe for HIV-positive individuals on ARVs [77]. Future ARV treatment, particularly in developing countries [78] will need to be scaled-up with treatment guidelines that include information on concomitant alcohol consumption. Physician and healthcare worker training with regard to patient management in this area will be necessary.

A South African pilot study by Morojele et al. [71] showed, contrary to expectations, that HIV health care providers do address the alcohol and HIV link and inform their clients thereof. The problem appears to be the lack of resources, information and skills required for adequate intervention.

A clear understanding of the relationship between ARVs and alcohol has important implications for better clinical management of patients. It is important to identify the knowledge gaps and suggest the type of studies that need to be undertaken to provide relevant data. The various mechanisms and pathways whereby alcohol impacts negatively for PLWA and particularly those on ART need to be consolidated further. These studies need to measure the impact of alcohol on HIV acquisition, re-infection and disease progression and how best to intervene to reduce the effect of alcohol in these three areas. An example of the latter is a recent study by Michel et al. [79]: The study identified specific groups of ART patients requiring targeted interventions for harmful alcohol consumption risk reduction. The objective was to promote improved HIV prevention and the effective use of ART in populations.

Hahn et al. [76] calls for more research examining the relationship between alcohol consumption and health service utilization for HIV testing and care. Studies have produced some conflicting results and further research relating alcohol to HIV testing and early linkage to care needs to be undertaken. Van Geertruyden et al. [29] recommend that the impact of alcohol use on ART adherence and sexual risk behavior has to be, documented urgently in sub-Saharan Africa and that consequently, intervention models need to be developed and evaluated.

HIV-positive individuals may not disclose drinking behaviour to health care workers, fearing they may be further stigmatised and that ART will be withheld. Improved measurement of alcohol exposure is important in order to understand the effect of alcohol on HIV outcomes better, hence improved measures of individual alcohol exposure is essential. Hahn et al. [8] recommend the use of sensitive and specific biologic markers that can address the under-reporting of alcohol consumption. Also devising methods that result in the full disclosure of alcohol consumption will also be helpful in this regard. The results from the study by Míguez-Burbano et al. [10] indicate that the type of alcohol consumed is of clinical relevance and needs to be considered in future studies.

Recently, it has been demonstrated, that compared with indicators of drinking frequency or pattern, the AUDIT measure has stronger associations with measures of variables such as inconsistent condom use and STI symptoms [80]. The use of the AUDIT may help further elucidate the relationship between alcohol consumption and sexual risk behaviour in future studies of alcohol and sexual risk behaviour. Kader et al. [81] conducted a preliminary investigation of the AUDIT and DUDIT in comparison to biomarkers for alcohol and drug use among HIV-infected clinic attendees in Cape Town, South Africa.

Notwithstanding the dearth of directed research on the interface of alcohol and HIV/AIDS, the following areas have been highlighted as important to follow-up:

These include the screening of HIV patients for alcohol problems and persons in treatment for alcohol problems need to be screened for HIV [82]. The use of interventions for alcohol problems as HIV prevention, by implementing established, effective interventions for alcohol problems [83]. The policy implications include the integration of HIV/AIDS and alcohol services [82]. Furthermore, there is a need to determine what levels of alcohol consumption, if any, are considered safe for HIV patients on ART. It is essential to acquire clear evidencebased guidelines on alcohol consumption for HIV-positive patients and their health care providers.

In the interim, it is recommended, where possible, that HIV and ART patients with identified problematic drinking behaviour should be monitored; the use of a biomarker is deemed unnecessary in a program environment [81]. There are many alcoholism screening tools that could be used to screen HIV-infected patients with regard to problematic alcohol use. These patients could then be engaged in an intervention most appropriate for their patterns and amounts of alcohol consumption in the context of their HIV infection. Furthermore, patients with identified problematic drinking behaviour should be educated on the dangers of the noxious combination of ARVs and alcohol and counselled to at least reduce alcohol consumption to prescribed levels, with the goal of abstention from alcohol consumption and adherence to prescribed ART regimens. It should also be emphasized that it is not advisable to stop taking ARVs to indulge in a binge drinking episode.

\section{Acknowledgement}

The preparation of this manuscript was funded by the US President's Emergency Fund for AIDS Relief (PEPFAR) through the US Centers of Disease Control and Prevention (CDC) (PO S-SF750-06-M-0781). Its contents are solely the responsibility of the authors and do not necessarily represent the official views of CDC or PEPFAR.

\section{References}

1. Rehm J, Rehn N, Room R, Monteiro M, Gmel G, et al. (2003) The global distribution of average volume of alcohol consumption and patterns of drinking. Eur Addict Res 9: 147-156.

2. UNAIDS, AIDS epidemic update (2009) “UNAIDS/09.36E / JC1700E”.UNAIDS World Health Organization.

3. Motlanthe K (2011) ARVs open to all. The Times, Sapa 12 August, 2011.

4. Motsoaledi A (2011) 'How we're re-engineering the health system „Motsoaledi: Health Budget Vote Policy Speech presented at the National Assembly.

5. Oh J, Hegele RA (2007) HIV-associated dyslipidaemia: pathogenesis and treatment. Lancet Infect Dis 7: 787-796.

6. Gmel G, Shield KD, Rehm J (2011) Developing a method to derive alcohol- attributable fractions for HIVIAIDS mortality based on alcohol's impact on adherence to antiretroviral medication. Popul Health Metr 9: 5.

7. Neblett RC, Hutton HE, Lau B, McCaul ME, Moore RD, et al. (2011) Alcoho consumption among HIV-infected women: impact on time to antiretroviral therapy and survival. J Women's Health (Larchmt) 20: 279-286.

8. Hahn JA, Bwana MB, Javors MA, Martin JN, Emenyonu NI, et al. (2010) Biomarker Testing to Estimate Under-Reported Heavy Alcohol Consumption by Persons with HIV Initiating ART in Uganda. AIDS Behav 14: 1265-1268.

9. World Health Organization (2011) Global Status Report on Alcohol and Health. Geneva.

10. Míguez-Burbano MJ, Lewis JE, Fishman J, Asthana D, Malow RM (2009) The influence of different types of alcoholic beverages on disrupting highly active antiretroviral treatment (HAART) outcome. Alcohol and Alcoholism 44: 366-371.

11. Baliunas D, Rehm J, Irving H, Shuper $P$ (2010) Alcohol consumption and risk 
Citation: Schneider M, Neuman M, Chersich M, Parry C (2011) Alcohol and Antiretroviral Therapy - A Lethal Cocktail. J AIDS Clinic Res S1:005. doi:10.4172/2155-6113.S1-005.

of incident human immunodeficiency virus infection: a meta-analysis. Alcohol consumption and risk of incident human immunodeficiency virus infection: a meta-analysis. Int J Public Health 55: 159-166.

12. Shuper PA, Neuman M, Kanteres F, Baliunas D, Joharchi N, et al. (2010) Causal considerations on alcohol and HIVIAIDS a systematic. Alcohol Alcohol 45: 159-166.

13. Braithwaite RS, Conigliaro J, Roberts MS, Shechter S, Schaefer A, et al. (2007) Estimating the impact of alcohol consumption on survival for HIV- individuals. AIDS Care 19: 459-466.

14. Kalichman SC (1999) Psychological and social correlates of high-risk sexual behaviour among men and women living with HIVIAIDS. AIDS Care 11: 415427.

15. Kalichman SC, Rompa D, Cage M (2000) Sexually transmitted infections among HIV seropositive men and women. Sex Transm Infect 76: 350-354.

16. Kalichman SC, Simbayi LC, Kaufman M, Cain D, Jooste S (2007) Alcohol use and sexual risks for HIVIAIDS in sub-Saharan Africa: systematic review of empirical findings. Prev Sci 8: 141-151.

17. Brennan DJ, Welles SL, Miner MH, Ross MW, Rosser BR, et al. (2010) HIV treatment optimism and unsafe anal intercourse among HIV-positive men who have sex with men: findings from the positive connections study. AIDS Educ Prev 22: 126-137.

18. Do NT, Phiri K, Bussmann H, Gaolathe T, Marlink RG, et al. (2010) Psychosocial factors affecting medication adherence among HIV-1 infected adults receiving combination antiretroviral therapy (cART) in Botswana. AIDS Res Hum Retroviruses 26: 685-691.

19. Andreani G, Espada C, Ceballos A, Ambrosioni J, Petroni A, et al. (2011) Detection of HIV-1 dual infections in highly exposed treated patients. Virol J 8: 392.

20. Wasserheit JN (1992) Epidemiological synergy. Interrelationships between human immunodeficiency virus infection and other sexually transmitted diseases. Sex Transm Dis 19: 61-77.

21. Theall KP, Amedee A, Clark RA Dumestre J, Kissinger $P$ (2008) Alcohol consumption and HIV-1 vaginal RNA shedding among women. J Stud Alcohol Drugs 69: 454-458.

22. Lima V, Harrigan R, Murray M, Moore D, Wood E, et al. (2008) Differential impact of adherence on long-term treatment response among naive HIV- infected individuals. AIDS 22: 2371-2380.

23. Gardner EM, Burma WJ, Steiner JF, Anderson PL, Bangsberg DR (2009) Antiretroviral medication adherence and the development of class-specific antiretroviral resistance. AIDS 23: 1035-1046.

24. Jaspan HB, Mueller AD, Myer L, Bekker LG, Orrell C (2011) Effect of Caregivers' Depression and Alcohol Use on Child Antiretroviral Adherence in South Africa. AIDS Patient Care STDS 25: 595-600.

25. Hendershot C, Stoner S, Pantalone, Simoni J (2009) Alcohol Use and Antiretroviral Adherence: Review and Meta-Analysis. J Acquir Immune Defic Syndr 52: $180-202$

26. Dahab M, Charalambous S, Hamilton R, Fielding K, Kielmann K, et al. (2008) "That is why I stopped the ART": Patients' \& providers' perspectives on barriers to and enablers of HIV treatment adherence in a South African workplace programme. BMC Public Health 8: 63.

27. Venkatesh KK, Srikrishnan AK, Mayer KH, Kumarasamy N, Raminani S, et al. (2010) Predictors of nonadherence to highly active antiretroviral therapy among HIV-infected South Indians in clinical care: implications for developing adherence interventions in resource-limited settings. AIDS Patient Care STDS 24: 795-803.

28. Jaquet A, Ekouevi DK, Bashi J, Aboubakrine M, Messou E, et al. (2010) Alcohol use and non-adherence to antiretroviral therapy in HIV-infected patients in West Africa. Addiction 105: 1416-1421.

29. Van Geertruyden JP, Woelk G, Mukumbi H, Ryder H, Colebunders R (2010) Alcohol and Antiretroviral Adherence? What About Africa? Letters to the Editor J Acquir Immune Defic Syndr 54: e10.

30. Díaz LE, Montero A, González-Gross M, Vallejo Al, Romeo J, et al. (2002) Influence of alcohol consumption on immunological status: a review. Eur $\mathrm{J}$ Clin Nutr 56: S50-S53.

31. Watzl B, Watson RR (1992) Role of alcohol abuse in nutritional immunosuppression. J Nutr 122: 733-737.
32. Balagopal A, Philip FH, Astemborski J, Block TM, Mehta A, et al. (2008) Human Immunodeficiency Virus-Related Microbial Translocation and Progression of Hepatitis C. Gastroenterology135: 226-233.

33. Molina PE, McClain C, Valla D, Guidot D, Diehl AM, et al. (2002) Molecula pathology and clinical aspects of alcohol induced tissue injury. Alcohol Clin Exp Res 26: 120-128.

34. Samet JH, Horton NJ, Traphagen ET, Lyon SM, Freedberg KA (2003) Alcoho consumption and HIV disease progression: Are they related? Alcohol Clin Exp Res 27: 862-867.

35. Samet JH, Cheng DM, Libman H, Nunes DP, Alperen JK, et al. (2007) Alcoho consumption and HIV disease progression. J Acquir Immune Defic Syndr 46: 194-199.

36. Fabris P, Tositti G, Manfrin V, Giordani MT, Vaglia A, et al. (2000) Does alcohol intake affect highly active antiretroviral therapy (HAART) response in HIVpositive patients? J AIDS 25: 92-93

37. Baum MK, Rafie C, Lai S, Sales S, Page JB, et al. (2010) Alcohol Use Accelerates HIV Disease Progression. AIDS Research and Human Retroviruses 26 511-518.

38. Goral J, Karavitis J, Kovacs EJ (2008) Exposure-dependent effects of ethanol on the innate immune system. Alcohol 42: 237-247.

39. Meadows GG, Wallendal M, Kosugi A, Wunderlich J, Singer DS (1992) Ethano induces marked changes in lymphocyte populations and natural killer cell activity in mice. Alcohol Clin Exp Res 16: 474-479.

40. Szabo G (1999) Consequences of alcohol consumption on host defence. Alcohol 34: 830-841.

41. National Institute on Alcohol Abuse and Alcholism (2006) Niaaa Plan for HivRelated Biomedical Research: Strenthening Alcohol and HIV / AIDS Biomedical Research. Report to the Extramural Advisory Board October 4-5.

42. Neuman MG, Monteiro M, Rehm J (2006) Drug Interactions between Psychoactive Substances and Antiretroviral Therapy in Individuals Infected With Human Immunodeficiency and Hepatitis Viruses. Subst Use Misuse 41: 1395-1463.

43. Neuman MG, Brenner DA, Rehermann B, Taieb J, Chollet-Martin S (2001) Mechanisms of alcoholic liver disease. Alcohol Clin Exp Res 25: 251S-253S.

44. Lieber CS (1978) Pathogenesis and early diagnosis of alcoholic liver injury. N Engl J Med 298: 888-893.

45. Lieber CS (2000) Hepatic, metabolic, and nutritional disorders of alcoholism from pathogenesis to therapy. Crit Rev Clin Lab Sci 37: 551-584.

46. Neuman MG, Shear NH, Cameron RG, Katz G, Tiribelli C (1999) Ethanol-Induced Apoptosis In Vitro. Clin Biochem 32: 547-555.

47. Parker WB, Cheng YC (1994) Mitochondrial toxicity of antiviral nucleoside analogs. J NIH RES 6: 57-61.

48. Lieber CS (2004) CYP2E1: from ASH to NASH. Hepatol Res 28: 1-11.

49. Miguez MJ, Shor-Posner G, Morales G, Rodriguez A, Burbano X (2003) HIV treatment in drug abusers: impact of alcohol use. Addict Biol 8: 33-37.

50. Zimmerman HJ (1999) Hepatotoxicity the adverse effects of drugs and other chemicals on the liver. (pp. 498-509) 2 ed. Philadelphia: Lippincott Williams and Wilkins.

51. Pandrea I, Happel KI, Amedee AM, Bagby GJ, Nelson S (2010) Alcohol's role in HIV transmission and disease progression. Alcohol Res Health 33: 203-218.

52. Jiménez-Nácher I, Alvarez E, Morello J, Rodriguez-Nóvoa S, de Andrés S, et al. (2011) Approaches for understanding and predicting drug interactions in human immunodeficiency virus-infected patients. Expert Opin Drug Metab Toxico 7: $457-477$.

53. DeLeve LD, Kaplowitz N (1991) Glutathione metabolism and its role in hepatotoxicity. Pharmac Ther 52: 287-305.

54. Buhl R, Jaffe HA, Holroyd KJ, Wells FB, Mastrangeli A, et al. (1989) Systemic glutathione deficiency in symptom-free HIV-seropositive individuals. Lancet 2 1294- 1298.

55. Staal FJ, Ela SW, Roederer M, Anderson MT, Herzenberg LA (1992) Glutathione deficiency and human immunodeficiency virus infection. Lancet 339: 909912. 
Citation: Schneider M, Neuman M, Chersich M, Parry C (2011) Alcohol and Antiretroviral Therapy - A Lethal Cocktail. J AIDS Clinic Res S1:005. doi:10.4172/2155-6113.S1-005.

56. Vogel M, Boesecke C, Wasmuth JC, Rockstroh JK (2010) HIV and hepatitis C co-infection. Dtsch Med Wochenschr 135: 1186-1191.

57. Romeo R, Rumi MG, Donato MF, Cargnel MA, Vigano P (2000) Hepatitis C is more severe in drug users with human immunodeficiency virus infection. J Viral Hepatitis 7: 297-301.

58. Prakash O, Mason A, Luftig RB, Bautista AP (2002) Hepatitis C virus (HCV) and human immunodeficiency virus type 1 (HIV-1) infections in alcoholics. Front Biosci 1: e286-300

59. Míguez-Burbano MJ, Lewis JE, Malow R (2009) "Alcohol and race/ethnicity elicit different changes in lipid profiles in HIV-infected individuals receiving highly active antiretroviral therapy". The J Assoc Nurses AIDS Care 20: 176-183.

60. Neuman MG, Malkiewicz IM, Phillips EJ, Rachlis A, Ong D, et al. (2002) Monitoring adverse drug reactions to sulphonamide antibiotics in human immunodeficiency virus infected individuals. Therapeutic Drug Monitoring 24: 728-736.

61. Fulco PP, Zingone MM, Higginson RT (2008) Possible Antiretroviral TherapyWarfarin Drug Interaction. Pharmacotherapy 28: 945-949.

62. Rehm J, Samokhvalov AV, Neuman MG, Room R, Parry C, et al. (2009) The association between alcohol use, alcohol use disorders and tuberculosis (TB). A systematic review. BMC Public Health 9: 450.

63. Bruce RD, Altice FL, Gourevitch MN, Friedland GH (2006) Pharmacokinetic drug interactions between opioid agonist therapy and antiretroviral medications: implications and management for clinical practice. J Acquir Immune Defic Syndr 41: 563-572.

64. Cvetkovic RS, Goa KL (2003) Lopinavir/ritonavir: a review of its use in the management of HIV infection. Drugs 63:769-802.

65. McCance-Katz EF, Rainey PM, Friedland G, Kosten TR, Jatlow P (2001) Effect of opioid dependence pharmacotherapies on zidovudine disposition. Am J Addict 10: 296-307.

66. Barber TJ, Marett B, Waldron S, Portsmouth S, Mackie NE, et al. (2007) Are disulfiram-like reactions associated with abacavir-containing antiretroviral regimens in clinical practice? AIDS 21: 1823-1824.

67. Sullivan LE, Saitz R, Cheng DM, Libman H, Nunes D, et al. (2008) The impact of alcohol use on depressive symptoms in human immunodeficiency virusinfected patients. Addiction 103: 1461-1467.

68. Leserman J (2008) Role of depression, stress, and trauma in HIV diseaseprogression. Psychosom Med 70: 539-545.

69. Chopra M, Kendall C, Hill Z, Schaay N, Nkonki LL, et al. (2006) "Nothing new": responses to the introduction of antiretroviral drugs in South Africa. AIDS 20: $1975-1977$.

70. Mogatle S, Skinner M, Mills E, Kanfer I (2008) Effect of African potato (Hypoxis hemerocallidea) on the pharmacokinetics of efavirenz. S Afr Med J 98: 945949 .

71. Morojele NK, Pithey AL, Kekwaletswe CT, Joubert-Wallis M, Pule MI, et al. (2010) South African health care providers recognition of the links between alcohol and HIV in their daily practice: a pilot study. African Journal of Drug and Alcohol Studies 9: 1-14.

72. Kalichman SC, Amaral CM, White D, Swetsze C, Howard P, et al. (2009) Prevalence and Clinical Implications of Interactive Toxicity Beliefs Regarding Mixing Alcohol and Antiretroviral Therapies among People Living with HIVIAIDS. AIDS Patient Care STDs 23: 449-454.

73. Fitzgerald M, Collumbien M, Hosegood V (2010) "No one can ask me 'Why do you take that stuff?"': men's experiences of antiretroviral treatment in South Africa. AIDS Care 22: 355-360.

74. Shacham E, Agbebi A, Stamm K, Overton ET (2011) Alcohol consumption is associated with poor health in HIV clinic patient population: a behavioral surveillance study. AIDS Behav 15: 209-213.
75. Sankar A, Wunderlich T, Neufeld S, Luborsky M, (2007) Sero-positive African Americans "Beliefs about Alcohol and Their Impact on Anti-retroviral Adherence. AIDS Behav 11: 195-203.

76. Hahn JA, Woolf-King SE, Muyindike W (2011) Adding Fuel to the Fire: Alcohol's Effect on the HIV Epidemic in Sub-Saharan Africa. Curr HIVIAIDS Rep 8: 172-180.

77. Bryant KJ (2006) Expanding Research on the Role of Alcohol Consumption and Related Risks in the Prevention and Treatment of HIVIAIDS. Substance Use \& Misuse 41: 1465-1507.

78. Delva W, Pretorius C, Temmerman M (2009) Is scaling up enough to curb the HIV epidemic in southern Africa? SAMJ 99: 638-639.

79. Michel L, Carrieri MP, Fugon L, Roux P, Aubin HJ, et al. (2010) Harmful alcoho consumption and patterns of substance use in HIV-infected patients receiving antiretrovirals (ANRS-EN12-VESPA Study): relevance for clinical management and intervention. AIDS Care 22: 1136-1145.

80. Luchters S, Geibel S, Syengo M, Lango D, Kingola N, et al. (2011 ) Use of AUDIT, and measures of drinking frequency and patterns to detect associations between alcohol and sexual behaviour in male sex workers in Kenya. BMC Public Health 11: 384.

81. Kader R, Seedat S, Koch, JR, Parry $\mathrm{CDH}$ (in press) A preliminary investigation of the AUDIT and DUDIT in comparison to biomarkers for alcohol and drug use among HIV-infected clinic attendees in Cape Town, South Africa. African Journal of Psychiatry.

82. Parry CDH, Rehm J, Morojele NK (2010) Is There A Causal Relationship Between Alcohol and HIV? Implications for Policy Practice and Future Research Journal of Drug and Alcohol Studies 9: 81-91.

83. Chersich MF, Rees HV, Scorgie F, Martin G (2009) Enhancing global contro of alcohol to reduce unsafe sex and HIV in sub-Saharan Africa. Global Health 5: 16 